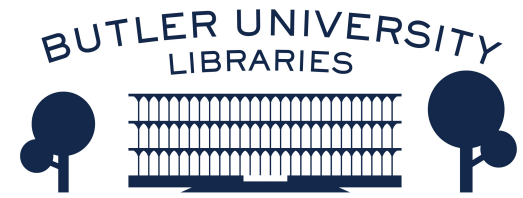

Butler University

Digital Commons @ Butler University

$10-21-2019$

Photometric and Spectroscopic Studies of V582 Lyr and V1016 Oph

Yao Cheng

Li-yun Zhang

Xianming L. Han

Liu Long

Hongpeng Lu

See next page for additional authors

Follow this and additional works at: https://digitalcommons.butler.edu/facsch_papers

Part of the Physics Commons 


\section{Authors}

Yao Cheng, Li-yun Zhang, Xianming L. Han, Liu Long, Hongpeng Lu, Qiang Yue, and Linyan Jiang 


\title{
Photometric and Spectroscopic Studies of V582 Lyr and V1016 Oph
}

\author{
Yao Cheng ${ }^{1}$, Li-yun Zhang ${ }^{1}$, Xianming L. Han ${ }^{1,2}$, Liu Long ${ }^{1}$, Hongpeng $\mathrm{Lu}^{1}$, Qiang Yue ${ }^{1}$, and Linyan Jiang ${ }^{1}$ \\ ${ }^{1}$ College of Science, Guizhou University, Guiyang 550025, People's Republic of China; liy zhang@ @otmail.com \\ ${ }^{2}$ Dept. of Physics and Astronomy, Butler University, Indianapolis, IN 46208, USA \\ Received 2018 October 13; revised 2019 August 31; accepted 2019 September 4; published 2019 October 21
}

\begin{abstract}
We present new CCD photometric light curves about two eclipsing binaries of V582 Lyr and V1016 Oph. Our observations were carried out by the SARA $91.4 \mathrm{~cm}$ telescope of America in 2016 and the $60 \mathrm{~cm}$ telescope of Chile in 2018. V582 Lyr's spectra type was classified as K5, and its radial velocity was determined using the LAMOST spectral survey. There are absorptions in the observed $\mathrm{H}_{\alpha}$ line and excess emissions in the subtracted $\mathrm{H}_{\alpha}$ line, which show weak chromospheric activity. We obtained the updated ephemeris information for V582 Lr and V1016 Oph, and found that

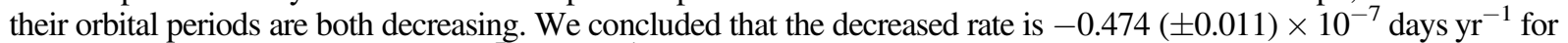
V582 Lyr and $3.460( \pm 0.014) \times 10^{-7}$ days $\mathrm{yr}^{-1}$ for V1016 Oph. For V582 Lyr, the period variation was interpreted as a mass transfer from the secondary component to the primary one, and the corresponding rate is $d M_{2} / d t=-1.10$ $( \pm 0.03) \times 10^{-7} M_{\odot} \mathrm{yr}^{-1}$. For V1016 Oph, we explain it by transferring from the primary component to the secondary one, and the corresponding rate is $d M_{1} / d t=-2.69( \pm 0.04) \times 10^{-7} M_{\odot} \mathrm{yr}^{-1}$. The photometric solution of V1016 Oph was obtained by analyzing the CCD photometry with the Wilson-Devinney program. We also obtained the orbital parameters of V1016 Oph by simultaneously analyzing our BVRI light curves and radial-velocity curve from the LAMOST low-resolution spectral survey. Finally, our orbital solution shows that they are contact eclipsing binaries with contact factors of $3.35( \pm 0.08) \%$ for V582 Lyr and $41.0( \pm 0.1) \%$ for V1016 Oph.
\end{abstract}

Key words: binaries: close - binaries: eclipsing - stars: individual (V582 Lyr, V1016 Oph)

Supporting material: machine-readable tables

\section{Introduction}

Eclipsing binaries are important in astrophysics and play a significant role in studying stellar parameters and evolution. Contact eclipsing binaries are a special kind of variable stars in which each component of a contact system has a larger surface than its Roche lobe according to Wilson's notation. They are synchronous circular orbit systems with common envelopes (Malkov et al. 2006). Some contact eclipsing binaries show long increases or decreases in period variation, which might be caused by mass transfer or angular momentum loss (Applegate 1992; Lanza et al. 1998; Zhu \& Qian 2006; etc.). The mass transfer causes the weak-contact binary into either a deepercontact or broken-contact configuration, according to thermal relaxation oscillation models (Lucy 1967; Robertson \& Eggleton 1977; Shaw 1994; etc.). It is interesting to study the properties of some new examples of contact binary.

V582 Lyr (2MASS J18553819+4058573, GSC 03123-01618, KIC 5685072) is a variable star, and was detected by Akerlof et al. (2000) in the ROTSE1 CCD survey. Blättler \& Diethelm (2000) obtained an unfiltered light curve and found its period was 0.2559007 days. The magnitude of V582 Lyr is from 10.904 to 14.429 and it is a W UMa eclipsing binary. Billings et al. (2001) obtained the full-phase light curve in the $\mathrm{V}$ single filter and updated its period to 0.25590067 (1) days. Recently, new minima times were published (e.g., Diethelm 2003, 2007a, 2010a, 2010b, 2012; Nelson 2011; Hübscher et al. 2012; Hübscher 2017; Lampens et al. 2017). They are also in Kepler eclipsing binary stars (Armstrong et al. 2014; Conroy et al. 2014; Kirk et al. 2016).

Original content from this work may be used under the terms of the Creative Commons Attribution 3.0 licence. Any further distribution of this work must maintain attribution to the author(s) and the title of the work, journal citation and DOI.
V1016 Oph was found to be a variable star in 1965 (Kinman et al. 1965; Kukarkin et al. 1968). The system was classified as a W-type contact eclipsing binary with a period of 0.407161 days (Avvakumova et al. 2013; Drake et al. 2014). Recently, new light minima were published (e.g., Krajci 2006; Diethelm 2011, 2012). It is important to derive minima times and orbital parameters for V1016 Oph.

We present four multi-band light curves of V582 Lyr and V1016 Oph. It is important to obtain their photometric solution based on the photometric observations and discuss their period variations.

\section{Photometric and Spectroscopic Observations}

The most direct method to obtain stellar information is photometric and spectroscopic observations. We obtained a set of charge-coupled device (CCD) photometric observation data for V582 Lyr in the BVRI bands on 2016 July 4 with the $91.4 \mathrm{~cm}$ telescope of the Southeastern Association for Research in Astronomy (SARA) at Kitt Peak National Observatory (Keel et al. 2017). The $B V R I$ filters are a Bessel system with an effective field of view of about $15^{\prime} \times 15^{\prime}$ and $1024 \times 1024$ CCD pixel. The limit magnitude with a signal-to-noise ratio $(\mathrm{S} / \mathrm{N})$ of 10 is about $20 \mathrm{mag}$ of the Vfilter in 10 minutes. The exposure times of V582 Lyr are $90 \mathrm{~s}$ in the $B$ band, $60 \mathrm{~s}$ in the $V$ band, and $30 \mathrm{~s}$ in the Rand $I$ bands. We chose to check and compare stars as follows: the magnitudes and color indexes of two or more stars are similar to those of our targets, and their positions are near those of our targets. After several runs, we finally chose TYC3123-1116-1 as our comparison star, and 2mass J18553073 +4100422 as the check star. Table 1 shows the coordinates and magnitudes in 2MASS JHK magnitudes (Cutri et al. 2003) for V582 Lyr, TYC3123-1116-1, and 2mass J18553073+4100422 from the web (http://simbad.u-strasbg.fr). We process these 
Table 1

The Observational Log of V582 Lyr and V1016 Oph, Comparison and Check Stars

\begin{tabular}{llcrrrr}
\hline \hline Targets & Name & Coordinates (R.A.; decl. 2000) & Mag_J & Mag_H & Mag_K & Source \\
\hline Variable star & V582 Lyr & $18: 55: 38.19 ;+40: 58: 57.4$ & 11.594 & 10.988 & 10.904 & $(1)$ \\
Comparison star & TYC 3123-1116-1 & $18: 55: 58.83 ;+40: 58: 29.4$ & 9.926 & 9.392 & 9.257 & $(1)$ \\
Check star & 2mass J18553073+4100422 & $18: 55: 30.74 ;+41: 00: 42.2$ & 13.298 & 13.051 & 12.951 & $(1)$ \\
\hline Variable star & V1016 Oph & $16: 16: 38.44 ;-05: 21: 18.1$ & 11.918 & 11.680 & 11.638 & $(1)$ \\
Comparison star & 2mass J16164050-0517035 & $16: 16: 40.50 ;-05: 17: 03.5$ & 11.154 & 10.565 & 10.451 & $(1)$ \\
Check star1 & 2mass J16163724-0517506 & $16: 16: 37.24 ;-05: 17: 50.6$ & 11.682 & 11.133 & 10.967 \\
Check star2 & 2mass J16164735-0523557 & $16: 16: 47.55 ;-05: 23: 55.7$ & 12.146 & 11.738 & 11.685 & $(1)$ \\
\hline
\end{tabular}

Note. (1) Cutri et al. (2003); The $J, H$, and $K s$ magnitudes are from the 2Mass survey.

photometric data with the $\mathrm{IRAF}^{3}$ package. The data reduction include bias subtraction, flat-fielding, cosmic-ray removal, and aperture photometry (Massey \& Davis 1992). The results of these light curves are plotted in Figure 1. The upper part of the figure shows the different magnitudes of our objects and the comparison star. The lower part shows the different magnitudes of the comparison and check stars, which are almost constant. The original photometric data of V582 Lyr were listed in Table 2. For V1016 Oph, we compiled CCD photometric observations from three nights (2018 April 23, April 27, and May 3) in the BVRI bands using the $0.6 \mathrm{~m}$ telescope of SARA at the Cerro Tololo Inter-American Observatory. The CCD resolution is also $1024 \times 1024$ pixels. The $B V R I$ filters are also a Bessel system with a $\sim 13^{\prime} \times 13^{\prime}$ field. The limit magnitude with an $\mathrm{S} / \mathrm{N}$ of 10 is about 19 mag of $V$ filter in 10 minutes (Keel et al. 2017). These images were processed using MaximDL. ${ }^{4}$ The uncertainties of V1016 Oph are $0.015 \mathrm{mag}$ for the $B$ filter, $0.011 \mathrm{mag}$ for the $V$ filter, $0.009 \mathrm{mag}$ for the $R$ filter, and 0.016 for the $I$ filter. The uncertainties of V582 Lyr $(0.003,0.004,0.005$, and $0.006 \mathrm{mag}$ for the BVRI filters, respectively) are better than those of V1016 Oph because of the better observational site and weather. Finally, the results of the $\Delta$ magnitude of light curves are plotted in Figure 1. We list the the data of V1016 Oph in Table 2.

The Large Sky Area Multi-Object Fibre Spectroscopic Telescope (LAMOST), also known as the GuoShoujing Telescope, is a meridian reflecting Schmidt and $4 \mathrm{~m}$ optical telescope for spectral survey (Wang et al. 1996; Cui et al. 2012). It obtained about 4000 spectra of objects with low resolutions of $\sim 1800 \AA$ and in the wavelength region range of 3700-9000 $\AA$ (Luo et al. 2015). The LAMOST spectral survey provides the opportunity to study the spectral properties of eclipsing binaries (Qian et al. 2017; Zhang et al. 2017, 2018). Spectroscopic observation of V582 Lyr was performed with the LAMOST survey (Cui et al. 2012) from 2013 to 2017. The observational log is listed Table 3 , and includes the observational data, phase, spectral type, the effective temperature, radial velocity, and surface gravity. The spectral type was determined to be K5 ( \pm 2$)$ (Luo et al. 2015). The fundamental parameters use automated methods and software (Wu et al. 2011a) via minimizing the residual between

\footnotetext{
IRAF is distributed by the National Optical Astronomy Observatories, which are operated by the Association of Universities for Research in Astronomy, Inc., under cooperative agreement with the National Science Foundation.

4 Maxlmdl 6 is a professional astronomical mapping software; the main functions include camera and telescope control, and astronomical image processing (George 2000) to correct biases, and dark and flat fields. We reduced all CCD images from the program Maxlm DL 6 and obtained their photometric magnitudes.
}
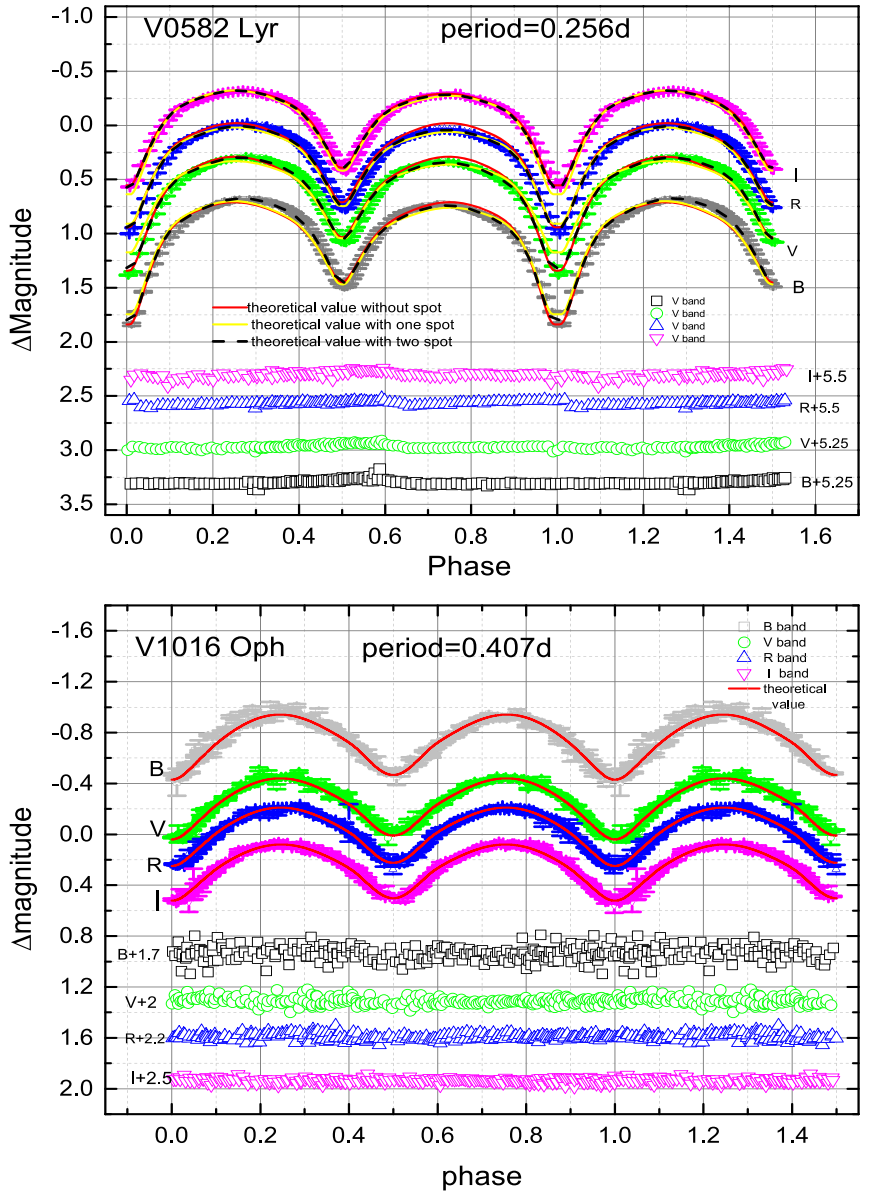

Figure 1. Light curves of V582 Lyr in the BVRI bands observed on 2016 July 04, and of V1016 Oph on three days (2018 April 23, 27 and May 3), with gray squares ( $\square$ ) for the $B$ band, green circles ( $\circ$ ) for the $V$ band, a blue triangle $(\triangle)$ for the $R$ band, and an inverted purple triangle $(\nabla)$ for the $I$ band. The magnitude difference between the comparison and check stars is in the lower panel. The red solid lines are the theoretical light curves for the spotless model, where the value of $\Sigma \omega_{i}(O-C)_{i}^{2}$ is 0.8444 for V582 Lyr and 2.1410 for V1016 Oph. The yellow solid lines are for the theoretical model with one spot, where the value of $\Sigma \omega_{i}(O-C)_{i}^{2}$ is 0.6380 for V582 Lyr, and the dashed black lines are for theoretical model light curves with two spots, where the value of $\sum \omega_{i}(O-C)_{i}^{2}$ is 0.2933 .

the observation and the model spectra, which are similar to ULySS (Koleva et al. 2009a, 2009b). The typical $1 \sigma$ error of the effect temperature obtained by LAMOST is about $167 \mathrm{~K}$. The uncertainty of the temperature of V582 Lyr is better than the typical value of $167 \mathrm{~K}$ for LAMOST spectra. We downloaded their spectroscopic data and plotted them in Figure 2. There are 
Table 2

Original Photometric Data of V582 Lyr Observed on 2016 July 4 and V1016 Oph Observed on 2018 April 23, 27, and May 3

\begin{tabular}{|c|c|c|c|c|c|c|c|c|}
\hline Object & HJD_B & $\Delta$ Mag_B & HJD_V & $\Delta$ Mag_V & HJD_R & $\Delta$ Mag_R & HJD_I & $\Delta$ Mag_I \\
\hline & d & 0.642 & d & mag & d & mag & $2 \mathrm{~d}$ & mag \\
\hline & 2457573.64726 & 0.642 & 2457573.64825 & 0.304 & 2457573.64889 & 0.017 & 2457573.64936 & -0.301 \\
\hline & 2457573.65023 & 0.691 & 2457573.65121 & 0.319 & 2457573.65185 & 0.021 & 2457573.65231 & -0.298 \\
\hline & 2457573.65317 & 0.698 & 2457573.65416 & 0.322 & 2457573.65480 & 0.019 & 2457573.65528 & -0.276 \\
\hline & 2457573.65613 & 0.697 & 2457573.65712 & 0.331 & 2457573.65777 & 0.032 & 2457573.65823 & -0.284 \\
\hline \multirow{9}{*}{$\begin{array}{l}\text { V582 Lyr } \\
2016 \text { Jul } 4\end{array}$} & 2457573.65908 & 0.727 & 2457573.66007 & 0.347 & 2457573.66070 & 0.043 & 2457573.66118 & -0.258 \\
\hline & $\ldots$ & $\ldots$ & $\ldots$ & $\ldots$ & $\ldots$ & $\ldots$ & $\ldots$ & $\ldots$ \\
\hline & 2457573.96633 & 1.286 & 2457573.96731 & 0.857 & 2457573.96795 & 0.518 & 2457573.96841 & 0.166 \\
\hline & 2457573.96928 & 1.202 & 2457573.97025 & 0.772 & 2457573.97089 & 0.442 & 2457573.97135 & 0.098 \\
\hline & 2457573.97222 & 1.125 & 2457573.97319 & 0.696 & 2457573.97383 & 0.381 & 2457573.97430 & 0.030 \\
\hline & $\cdots$ & $\cdots$ & 2458231.61223 & -0.437 & 2458231.61316 & -0.162 & 2458231.61391 & 0.081 \\
\hline & 2458231.61025 & -0.941 & 2458231.61631 & -0.358 & 2458231.61723 & -0.199 & 2458231.61798 & 0.063 \\
\hline & 2458231.61433 & -0.851 & 2458231.62039 & -0.410 & 2458231.62132 & -0.175 & 2458231.62207 & 0.112 \\
\hline & 2458231.61842 & -0.768 & 2458231.62448 & -0.403 & 2458231.62540 & -0.149 & 2458231.62616 & 0.088 \\
\hline \multirow{11}{*}{$\begin{array}{l}\text { V1016 Oph } \\
2018 \text { Apr } 23\end{array}$} & 2458231.62251 & -0.768 & 2458231.62892 & -0.389 & 2458231.62985 & -0.075 & 2458231.63059 & 0.095 \\
\hline & $\ldots$ & $\cdots$ & $\ldots$ & $\cdots$ & $\ldots$ & $\cdots$ & $\ldots$ & $\cdots$ \\
\hline & 2458231.92309 & -0.585 & 2458231.92508 & -0.105 & 2458231.92601 & 0.112 & 2458231.92676 & 0.394 \\
\hline & 2458231.92718 & -0.654 & 2458231.92917 & -0.171 & 2458231.93000 & 0.072 & 2458231.93085 & 0.333 \\
\hline & 2458231.93125 & -0.673 & 2458231.93325 & -0.182 & 2458231.93418 & 0.016 & 2458231.93493 & 0.310 \\
\hline & 2458231.93533 & -0.621 & 2458231.93733 & -0.228 & 2458231.93825 & 0.006 & 2458231.93899 & 0.267 \\
\hline & $\ldots$ & $\ldots$ & 2458231.94141 & -0.258 & 2458231.94233 & -0.037 & 2458231.94308 & 0.233 \\
\hline & 2458235.60002 & -0.653 & 2458235.60187 & -0.228 & 2458235.60279 & -0.228 & 2458235.60354 & 0.278 \\
\hline & 2458235.60468 & -0.697 & 2458235.60615 & -0.235 & 2458235.60707 & -0.235 & 2458235.60782 & 0.243 \\
\hline & 2458235.60897 & -0.712 & 2458235.61024 & -0.239 & 2458235.61116 & -0.239 & 2458235.61119 & 0.213 \\
\hline & 2458235.61305 & -0.739 & $\ldots$ & $\ldots$ & 2458235.61525 & -0.293 & 2458235.61600 & 0.217 \\
\hline \multirow{8}{*}{$\begin{array}{l}\text { V1016 Oph } \\
2018 \text { Apr } 27\end{array}$} & 2458235.61722 & -0.762 & 2458235.61848 & -0.293 & 2458235.61940 & -0.340 & 2458235.62015 & 0.197 \\
\hline & $\ldots$ & $\cdots$ & $\ldots$ & $\cdots$ & $\ldots$ & $\cdots$ & $\ldots$ & $\ldots$ \\
\hline & 2458235.92828 & -0.746 & 2458235.92955 & -0.322 & 2458235.93047 & -0.091 & 2458235.93122 & 0.243 \\
\hline & 2458235.93236 & -0.707 & 2458235.93363 & -0.222 & 2458235.93455 & 0.002 & 2458235.93531 & 0.285 \\
\hline & 2458235.93643 & -0.703 & 2458235.93771 & -0.221 & 2458235.93863 & 0.009 & 2458235.93937 & 0.268 \\
\hline & 2458235.94049 & -0.926 & 2458235.94177 & -2.513 & 2458235.94269 & 0.016 & 2458235.94343 & 0.315 \\
\hline & 2458235.94457 & -0.783 & 2458235.94584 & -0.401 & 2458235.94677 & 0.055 & 2458235.94752 & 0.357 \\
\hline & $\ldots$ & $\cdots$ & $\ldots$ & $\cdots$ & $\ldots$ & $\cdots$ & $\ldots$ & $\cdots$ \\
\hline \multirow[t]{3}{*}{2018 May 03} & 2458241.91324 & -0.696 & 2458241.91451 & -0.228 & 2458241.91544 & 0.015 & 2458241.91620 & 0.297 \\
\hline & 2458241.91733 & -0.692 & 2458241.91859 & -0.249 & 2458241.91952 & -0.025 & 2458241.92028 & 0.254 \\
\hline & $\ldots$ & $\cdots$ & $\ldots$ & $\ldots$ & $\ldots$ & $\ldots$ & $\ldots$ & $\ldots$ \\
\hline
\end{tabular}

(This table is available in its entirety in machine-readable form.)

Table 3

The Spectroscopic Parameters of the LAMOST Survey of V582 Lyr and Its Equivalent Width in the $\mathrm{H}_{\alpha}$ Line

\begin{tabular}{|c|c|c|c|c|c|c|c|}
\hline Date & HJD & Phase & Subtracted spectra: $\mathrm{H}_{\alpha}$ & Radial velocity & Spectra type & Temperature & $\log (g)$ \\
\hline $\mathrm{d}$ & d & & (A) & $\left(\mathrm{km} \mathrm{s}^{-1}\right)$ & $\cdots$ & (K) & $\cdots$ \\
\hline 2013 Oct 07 & 2456572.78967 & 0.21616 & $0.581 \pm 0.006$ & $17.49 \pm 13.64$ & K5 & $4595 \pm 79$ & 4.283 \\
\hline 2015 Oct 04 & 2457299.80939 & 0.19116 & $1.158 \pm 0.094$ & $21.27 \pm 12.15$ & K5 & $4642 \pm 39$ & 4.448 \\
\hline 2016 May 12 & 2457521.15146 & 0.12959 & $1.135 \pm 0.049$ & $17.24 \pm 15.93$ & K5 & $4617 \pm 39$ & 4.494 \\
\hline 2017 Jun 03 & 2457908.13599 & 0.34908 & $\ldots$ & $-17.98 \pm 7.53$ & K5 & $4550 \pm 43$ & 4.355 \\
\hline 2017 Jun 07 & 2457912.12620 & 0.94163 & $0.758 \pm 0.038$ & $-14.93 \pm 12.09$ & K5 & $4712 \pm 34$ & 4.478 \\
\hline
\end{tabular}

strong absorptions in the $\mathrm{H}_{\alpha}$ line. We observed V1016 Oph with the Lijiang $2.4 \mathrm{~m}$ telescope at Yunnan Astronomical Observatory, China on 2019 March 12th, with an exposure time of $900 \mathrm{~s}$. We used low-resolution Grism 3 and a long-slit width of 2!"5 covering the 3200-9200 $\AA$ wavelength range (Fan et al. 2015), which provides a resolution of $172 \AA / \mathrm{mm}$. The resolution is about 1077. We performed it by bias subtraction, flat-field calibration, cosmic-ray removal, and spectral extraction using the IRAF. We also plotted the spectrum of V1016 Oph and the library spectrum with spectral types G0, G5, and K0 from Pickles (1998) in Figure 3. Through comparison with their spectra, we find thespectral type of V1016 Oph is about G8 $( \pm 2)$. 

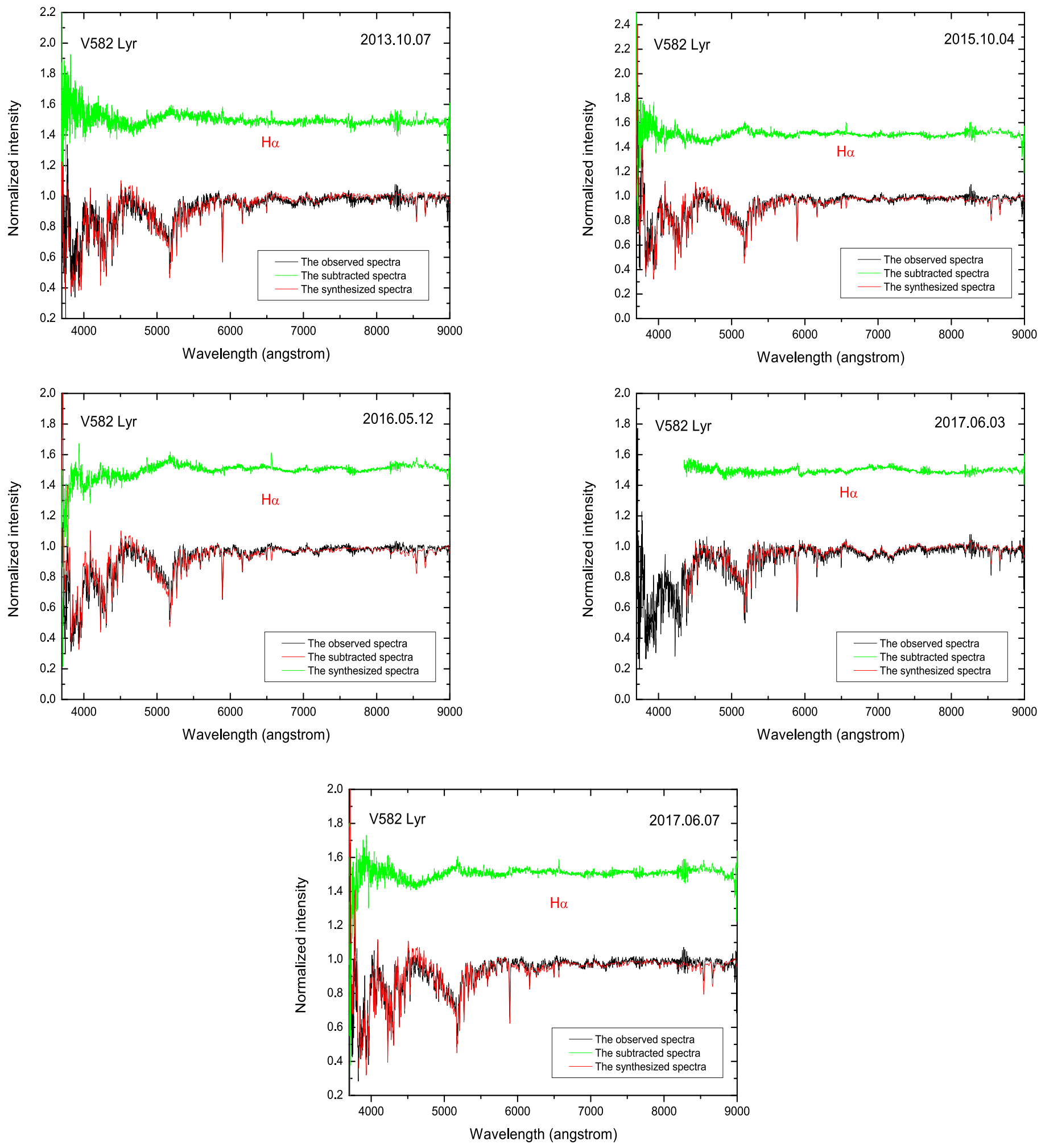

Figure 2. Five observed and synthesized spectra of V582 Lyr from the LAMOST survey in the $\mathrm{H}_{\alpha}$ line in 2013-2017.

\section{Period Analyses}

We selected the symmetric light curves with small phase intervals of about 0.05 near the primary and secondary minima to calculate light minima. We figured out the new minima times of V582 Lyr and V1016 Oph using the polynomial fitting program developed by Nelson (2014) with the method of Kwee \& van Woerden (1956) from our photometric observation data.
We provide a example for determining the minima times that show the photometry and the polynomial fits in Figure 4. We list the individual minima times, the weighted averages, and the uncertainties in the BVRI bands in Table 4. We also collected all the available light minima times of V582 Lyr and V1016 Oph from Eclipsing Binaries Minimum Times Database (http://var2.astro.cz/ocgate/) and past observations (including 


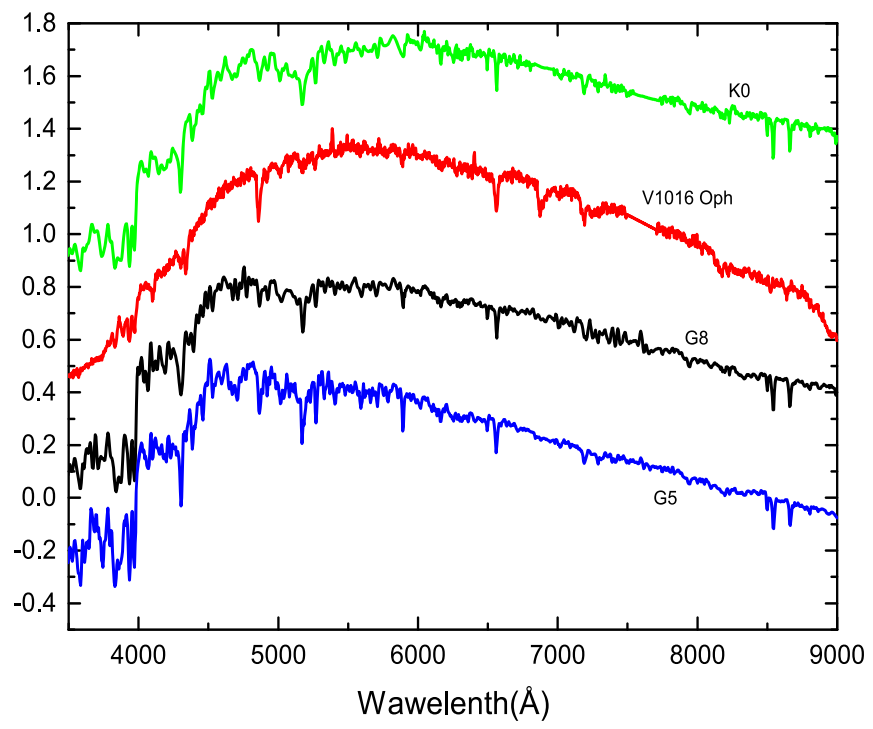

Figure 3. Middle line: the low-dispersion spectrum of V1016 Oph observed by Lijiang 2.4 m telescope on 2019 March 12. The others are K0, G8 and G5 from the Pickles (1998).

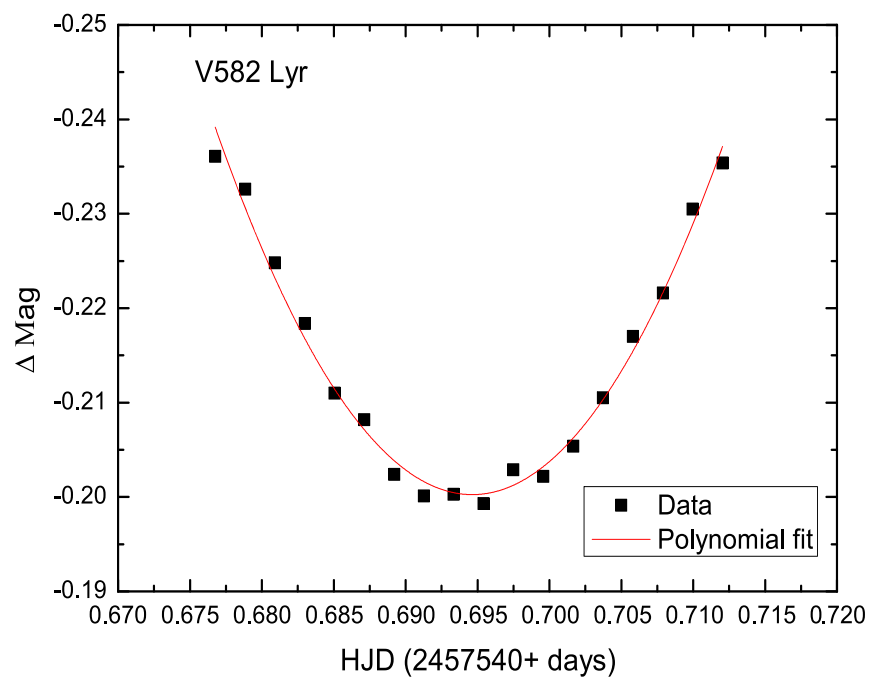

Figure 4. Example determination of the minima times using the polynomial fitting. The circles are the photometry and the red line is the polynomial fits.

all Kepler minima) in the literature (e.g., Blättler \& Diethelm 2000; Billings et al. 2001; Diethelm 2003; Conroy et al. 2014). These are listed in Table 5. The additional 1500 consecutive minima times from Kepler are the ideal data set for computing a period variation. We updated their ephemeris and analyze the periodic variations of our objects.

\subsection{Period Analysis of V582 Lyr}

Usually, the minima of eclipsing binaries are obtained by an optical telescope with an aperture smaller than $1 \mathrm{~m}$. There are different observational methods for the relative flux of the light curve (Vis is from visual observation and CCD is a chargecoupled device) to determine the light minima. Because of the differing amounts of measurements across minima, we assigned weighting factors of 1 and 10 to Vis and CCD data, respectively. The weight factors have been used in the analyses of other eclipsing binaries (Yang et al. 2009). We calculated the new linear ephemeris and quadratic ephemeris by the least- squares method with the error-weighted. If some minima times have no uncertainties in the literature, we used the mean uncertainties of other data with the same observational method:

$$
\begin{aligned}
\text { Min. I }= & \text { HJD2451281.9010 }( \pm 0.0006) \\
& +0.25590483( \pm 0.00000005) E,
\end{aligned}
$$

where 2451281.9012 is set to the initial time, 0.25590475 days is the value of the new, more accurate orbital period we found, and $E$ is epoch number; the decimal in parentheses indicates the error. The $(O-C) \mathrm{I}$ values of linear fit residuals are listed in the seventh column of Table 5. The values of $(O-C)$ I are shown in Figure 5 to clarify its period variation of V582 Lyr; a downward curve is visible. The quadratic ephemeris is

$$
\begin{aligned}
\text { Min. I }= & \text { HJD2451281.8993 }\left( \pm e^{-10.0}\right)+0.25590517 \\
& \times\left( \pm e^{-20.5}\right) E-0.152\left( \pm e^{-7.7}\right) \times 10^{-10} E^{2},
\end{aligned}
$$

where $-0.1152( \pm 0.003) \times 10^{-10}$ in Equation (2) is the quadratic coefficient, which means that its period decreases and the rate is $0.474( \pm 0.011) \times 10^{-7}$ days $\mathrm{yr}^{-1}$.

\subsection{Period Analysis of V1016 Oph}

For V1016 Oph, we used the same method to collect the light minimum times that are listed in Table 5. We fit the result with new data from light minimum times from our observation. Considering the various measurement accuracies, using the same method, the new ephemeris became

$$
\begin{aligned}
\text { Min. I }= & \text { HJD2448447.4455 }( \pm 0.0030) \\
& +0.40716086( \pm 0.0000003) E,
\end{aligned}
$$

where 2448447.4455 is the initial epoch, 0.40716086 days is the number of new, more accurate orbital periods, and the epoch number is $E$. We provide the residual $(O-C) \mathrm{I}$ of linear fitting in the seventh column of Table 5. Likewise, the values of $(O-C) \mathrm{I}$ are shown in Table 5 to clarify the period variation and a clear downward parabolic curve. The quadratic ephemeris we obtained is

$$
\begin{aligned}
\operatorname{Min} . \mathrm{I}= & H J D 2448447.4499\left( \pm e^{-7.6}\right)+0.40716404 \\
& \times\left( \pm e^{-17.3}\right) E-0.193\left( \pm e^{-6.2}\right) \times 10^{-9} E^{2}
\end{aligned}
$$

where $-0.193( \pm 0.002) \times 10^{-19}$ in Equation (4) is a quadratic coefficient, which signifies that its orbital period is also decreasing and the reduced ratio is $0.346( \pm 0.001) \times 10^{-6}$ days $\mathrm{yr}^{-1}$.

\section{Orbital Parameters}

We observed several sets of CCD photometric data and thus obtained BVRI band light curves. We calculated the observational photometric data to deduce the solution of V582 Lyr and V1016 Oph.

We tried to obtain a photometric solution with the WilsonDevinney program (Wilson \& Devinney 1971; Wilson 1979, 1990, 1994; Wilson \& Van Hamme 2010, etc.). First, wedetermine which mode should be used in this program. We selected contact binary model in the program because V582 Lyr has been identified as an eclipsing binary star of EW type (Otero et al. 2006). We averaged the mean temperature 4623 $( \pm 47) \mathrm{K}$ as the initial value from the LAMOST (http://dr6. lamost.org/) spectra stellar parameter pipeline (Wu et al. 
Table 4

Newly Obtained Minima Times of V582 Lyr and V1016 Oph in the BVRI Bands

\begin{tabular}{|c|c|c|c|c|c|}
\hline HJD $(B)$ & HJD $(V)$ & $\operatorname{HJD}(R)$ & HJD $(I)$ & HJD (average) & Type \\
\hline $2457573.70151 \pm 0.00187$ & $2457573.70163 \pm 0.00164$ & $2457573.70132 \pm 0.00212$ & $2457573.70147 \pm 0.00182$ & $2456986.25742 \pm 0.00186$ & secondary \\
\hline $2457573.82908 \pm 0.00485$ & $2457573.82932 \pm 0.00437$ & $2457573.82946 \pm 0.00287$ & $2457573.82920 \pm 0.00324$ & $2457573.82927 \pm 0.00383$ & primary \\
\hline $2457573.95766 \pm 0.00101$ & $2457573.95750 \pm 0.00156$ & $2457573.95760 \pm 0.00237$ & $2457573.95768 \pm 0.00245$ & $2457573.95761 \pm 0.00185$ & secondary \\
\hline $2458231.69103 \pm 0.00435$ & $2458231.69675 \pm 0.00096$ & $2458231.69743 \pm 0.00049$ & $2458231.69765 \pm 0.00079$ & $2458231.69572 \pm 0.00165$ & secondary \\
\hline $2458231.89950 \pm 0.04144$ & $2458231.90164 \pm 0.00655$ & $2458231.90203 \pm 0.00170$ & $2458231.90232 \pm 0.00768$ & $2458231.90137 \pm 0.01434$ & primary \\
\hline $2458235.76852 \pm 0.02962$ & $2458235.76881 \pm 0.00416$ & $2458235.76912 \pm 0.01362$ & $2458235.76435 \pm 0.00947$ & $2458235.76770 \pm 0.01422$ & secondary \\
\hline $2458241.67140 \pm 0.00077$ & $2458241.67229 \pm 0.00037$ & $2458241.67320 \pm 0.00048$ & $2458241.67248 \pm 0.00026$ & $2458241.67234 \pm 0.00047$ & primary \\
\hline $2458241.87535 \pm 0.00149$ & $2458241.87618 \pm 0.00070$ & $2458241.87636 \pm 0.00028$ & $2458241.87595 \pm 0.00032$ & $2458241.87596 \pm 0.00070$ & secondary \\
\hline
\end{tabular}


Table 5

Minimum Times and Their Relevant Parameters for V582 Lyr and V1016 Oph

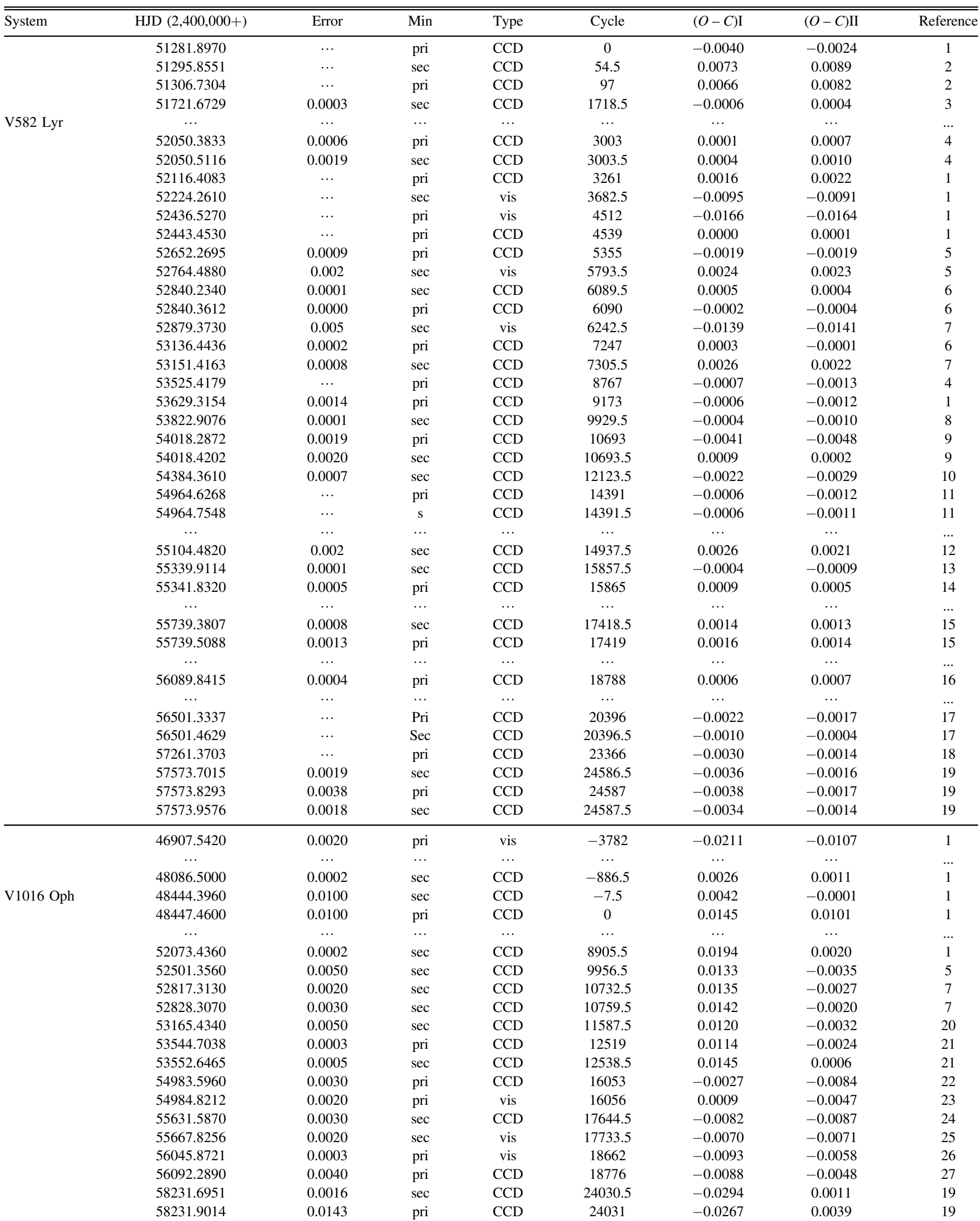


Table 5

(Continued)

\begin{tabular}{ccccccccc}
\hline \hline System & HJD $(2,400,000+)$ & Error & Min & Type & Cycle & $(O-C)$ I & $(O-C)$ II & Reference \\
\hline & 58235.7677 & 0.0142 & sec & CCD & 24040.5 & -0.0284 & 0.0022 & 19 \\
& 58241.6723 & 0.0005 & pri & CCD & 24055 & -0.0276 & 0.0030 & 19 \\
& 58241.8760 & 0.0007 & sec & CCD & 24055.5 & -0.0275 & 0.0032 & 19 \\
\hline
\end{tabular}

Note. Paschke \& Brát (2006); 2. Blättler \& Diethelm (2000); 3. Billings et al. (2001) 4. Brát et al. (2007) 5. Diethelm (2003) 6. Krajci (2005) 7. Diethelm (2004) 8. Nelson (2007) 9. Diethelm (2007a); 10. Diethelm (2007b); 11. Conroy et al. (2014): 12. Diethelm (2010a) 13. Nelson (2011) 14. Diethelm (2010b) 15. Hübscher et al. (2012); 16. Diethelm (2012) 17. Lampens et al. (2017) 18. Hübscher (2017); 19. Our paper; 20. Diethelm (2005) 21. Krajci (2006) 22. Paschke (2009) 23. Diethelm (2009) 24. Paschke (2012) 25. Diethelm (2011) 26. Diethelm (2012) 27. Paschke (2013).

(This table is available in its entirety in machine-readable form.)
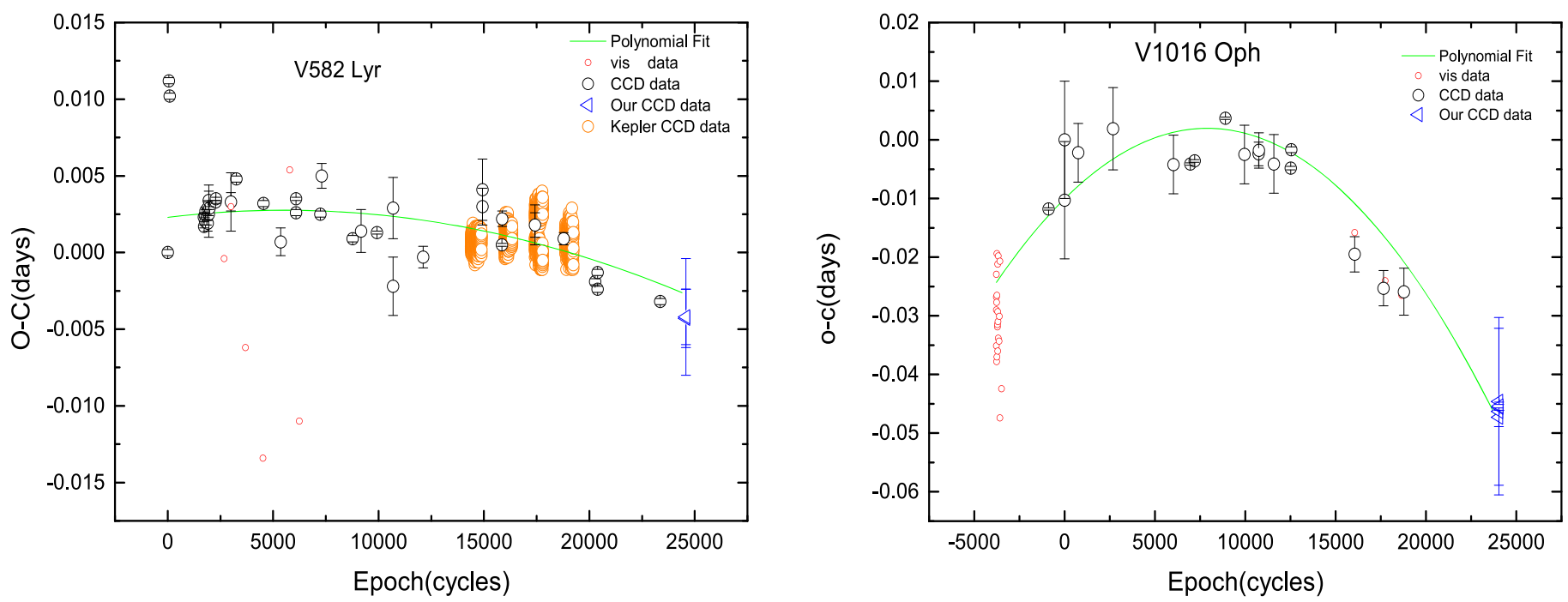

Figure 5. $(O-C)$ diagrams for V582 Lyr and V1016 Oph, where the initial time of V582 Lyr is HJD 2451281.8970 and that for V1016 Oph is 2448447.4600 . The small red circles $(\circ)$ are vis data, the black circles $(\circ)$ are CCD data, and the blue triangle $(\triangleleft)$ is our CCD data. The green and solid lines represent the polynomial fit.

2011b; Luo et al. 2015) in Table 2. This was determined based on the Universite de Lyon spectroscopic analysis software (ULySS; Koleva et al. 2009a, 2009b; Wu et al. 2011b), which was the initial value of the primary temperature. The fixed parameters in the Wilson-Devinney program were set as follows: the bolometric albedo $A_{1}=A_{2}=0.5$ (Rucinski 1973) and gravity-darkening coefficients $g_{1}=g_{2}=0.32$ (Lucy 1967). The bolometric limb-darkening coefficients $X_{\text {bolo }}$ and limbdarkening coefficients for the $B V R I$ bands are $X_{1 \text { bolo }}=0.533$, $X_{\text {bbolo }}=0.505 ; X_{1 B}=0.938, X_{2 B}=0.940, X_{1 V}=0.790, X_{2 V}$ $=0.794, X_{1 R}=0.653, X_{2 R}=0.666, X_{1 I}=0.529, X_{2 I}=0.532$. These values are interpolated based on the stellar temperature using the table from Van Hamme (1993).

Before adjusting the parameter, we should first determine an appropriate mass ratio $(q)$ value. We simultaneously analyzed the new light curves and one set of radial velocity from lowresolution spectra published by the LAMOST survey to obtain the best solution. Radial-velocity data are fed into the W-D program. Figure 6 is a plot of the results of that W-D program. The observed and theoretical radial-velocity curves are plotted in Figure 6, which also contains the radial velocity of the secondary component of V582 Lyr. As there is one set of radial-velocity curves and the data are very limited, we also search for a reliable mass ratio using the relation between $q$ and the residual. We search the more likely mass ratio ranges of $0.4-4$ with a step if about 0.2 from the $q$-search method of the W-D program. We calculated the results for different mass ratios $(0.4,0.5,0.6,0.8,1.0,1.2,1.3,1.4,1.5,1.6,1.7,1.8,1.9$,

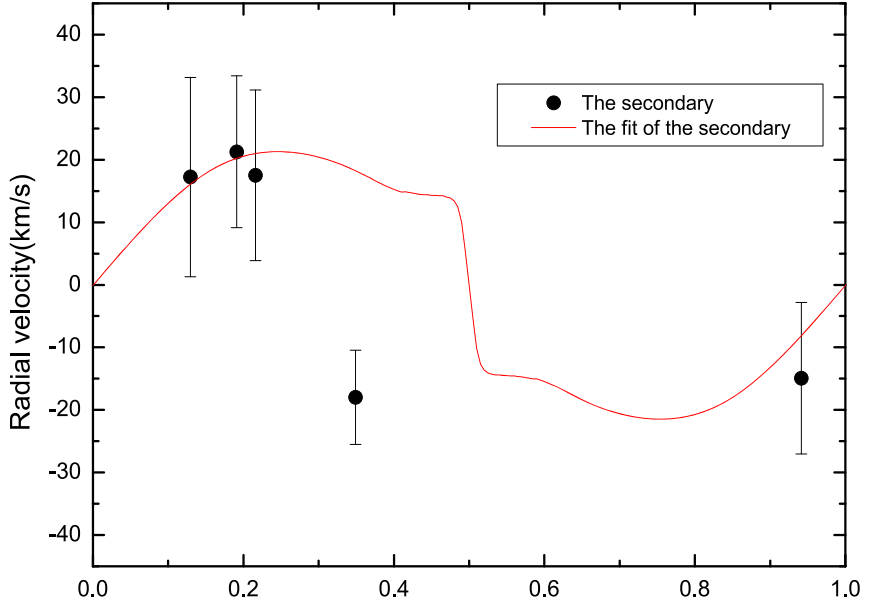

Figure 6. Radial velocities of the secondary components and their fits for V582 Lyr.

2.0, 2.2, 2.4, 2.6, 2.8, 3.0, .2, 3.4, 3.6, 3.8, 4.0). First, we fixed one of the mass ratios and simultaneously adjusted the other parameters. After many runs and many times, we obtained all the results of different mass ratios. It is a perfect method for using the physics of eclipsing binaries (Prsa \& Zwitter 2005; Prsa et al. 2016) or MCMC (Boffin et al. 2018). These fittings of $\Sigma \omega_{i}(O-C)_{i}^{2}-q$ are plotted in Figure 7. The most likely mass ratio is the corresponding value of the lowest residual. From Figure 7, after a lot of runs, we finally found the minimal 

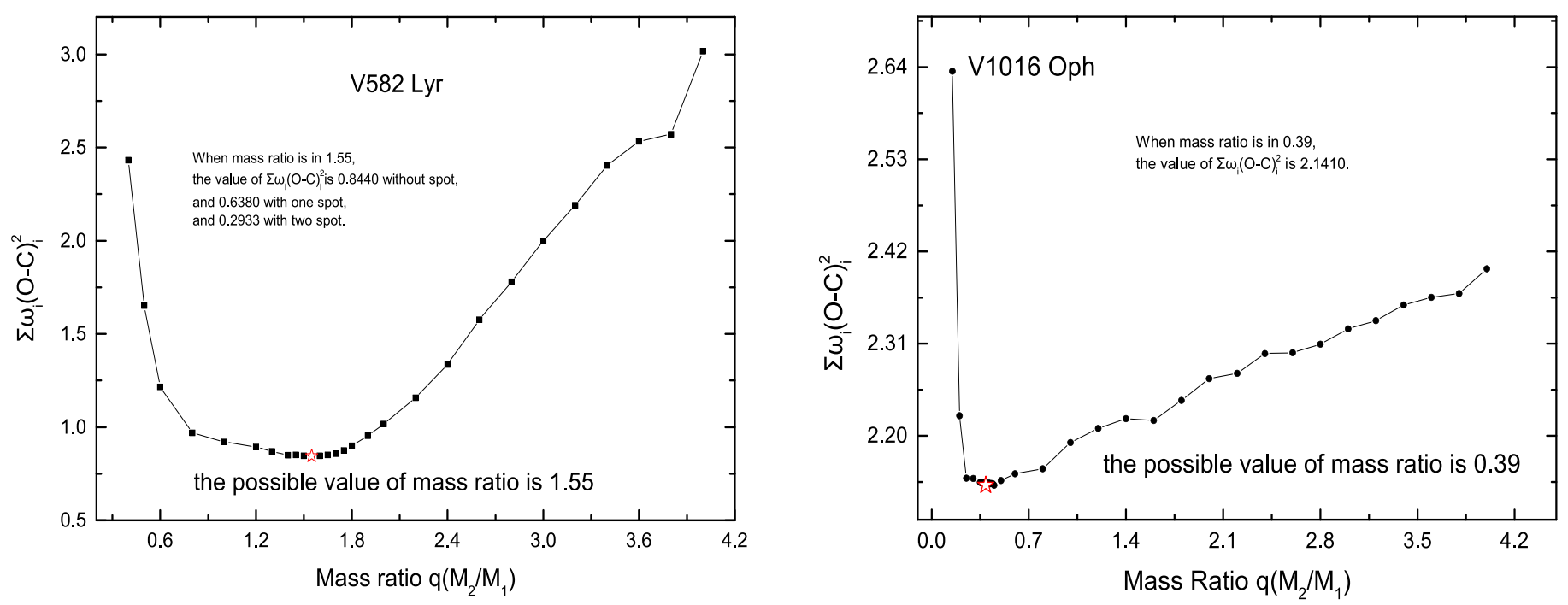

Figure 7. Relationships between $\Sigma \omega_{i}(O-C)_{i}^{2}$ and the mass ratios $q$ for V582 Lyr and V1016 Oph. The black line shows the connections between two points.

$\Sigma \omega_{i}(O-C)_{i}^{2}$ from the W-D program when the ratio $q=$ 1.55 , which means 1.55 is the likely mass ratio, where the value of $q$ is the mass of the secondary component relative to its primary. Because there are flux ratios from the light curves in multiple optical photometric bands at all orbital phases, we also use those to estimate the component temperatures. Moreover, we also adjust other parameters: orbital inclination $(i)$, mean temperatures of the primary and secondary components $\left(T_{1}\right.$ and $\left.T_{2}\right)$, primary component monochromatic luminosity $\left(L_{1 B}, L_{1 V}\right.$, $L_{1 R}$ and $\left.L_{1 I}\right)$, and dimensionless potentials $\left(\Omega_{1}=\Omega_{2}\right)$. For each parameter, we used the larger region of these parameters with different steps to search for the best solution. Finally, we found the final photometric solution of V582 Lyr after these adjustable parameters converged, which means that the corrections for the input parameters given by the W-D program are smaller than their standard deviations. The corresponding parameters are listed in Table 6. The photometric solution explains these BVRI photometric light curves. The theoretical and observed light curves are shown in Figure 1. Moreover, we present the corresponding stellar structure of our photometric solution in Figure 8.

According to the light-curve asymmetry and the fit of the observational data and theoretical light curves with no spot, we first add one spot on the primary component. The preliminary value of the spot longitude is determined from the centers of the depression of LCs. Because the spot temperature is roughly $1000 \mathrm{~K}$ below the photosphere, we set the temperature factor (Tspot/Tstar) as 0.8. We estimate the radius of the spot using the phase ranges of the light-curve distortion. We adjust the orbital parameters and spot parameters for the best result; for more details see Zhang \& Gu (2008). Because the fit between the observational data and the theoretical light curve is not very good using one spot (Figure 1), we have to add one more spot to explain it. Although the area of the spot is correlated with its temperature and the latitude of the spot is correlated with its radius. we also try our best to determine the spot parameters for the best fit, as our observations are in four colors. We assume the latitude $=90^{\circ}$ thanks to the degeneration. We obtained a two-spot model in our solution. The first spot parameters are as follows: latitude: $90^{\circ}$ a; longitude: $130^{\circ} .3 \pm 3^{\circ} .0$; radius: $27^{\circ} .0$ $\pm 5^{\circ} .2$; temperature: $5140 \pm 200 \mathrm{~K}$. The other spot's parameters are: latitude: $90^{\circ}$ a; longitude: $346^{\circ} .5 \pm 2^{\circ} .0$; radius: $21^{\circ} .3 \pm 6^{\circ} .7$;
Table 6

Theoretical Orbital Parameters of V582 Lyr and V1016 Oph

\begin{tabular}{|c|c|c|}
\hline & V582 Lyr & V1016 Oph \\
\hline Parameters & Values & Values \\
\hline$T_{1}(\mathrm{~K})$ & $4624 \pm 47$ & $5310 \pm 160$ \\
\hline$q\left(M_{2} / M_{1}\right)$ & $1.55 \pm 0.05$ & $0.39 \pm 0.01$ \\
\hline$T_{2}(\mathrm{~K})$ & $4366 \pm 20$ & $5260 \pm 20$ \\
\hline$i(\circ)$ & $87.5 \pm 0.4$ & $71.0 \pm 0.2$ \\
\hline$\Omega_{\text {in }}$ & 4.600 & 2.658 \\
\hline$\Omega_{\text {out }}$ & 4.020 & 2.419 \\
\hline$\Omega_{1}=\Omega_{2}$ & $4.588 \pm 0.007$ & $2.560 \pm 0.005$ \\
\hline$L_{1} /\left(L_{1}+L_{2}\right)(B)$ & $0.5221 \pm 0.0012$ & $0.7135 \pm 0.0013$ \\
\hline$L_{1} /\left(L_{1}+L_{2}\right)(V)$ & $0.4968 \pm 0.0011$ & $0.7099 \pm 0.0011$ \\
\hline$L_{1} /\left(L_{1}+L_{2}\right)(R)$ & $0.4746 \pm 0.0010$ & $0.7067 \pm 0.0010$ \\
\hline$L_{1} /\left(L_{1}+L_{2}\right)(I)$ & $0.4590 \pm 0.0007$ & $0.7040 \pm 0.0009$ \\
\hline$r_{1}($ pole $)$ & $0.321 \pm 0.001$ & $0.454 \pm 0.001$ \\
\hline$r_{1}($ side $)$ & $0.336 \pm 0.001$ & $0.489 \pm 0.001$ \\
\hline$r_{1}($ back $)$ & $0.369 \pm 0.001$ & $0.524 \pm 0.002$ \\
\hline$r_{1}$ (average) & $0.342 \pm 0.001$ & $0.489 \pm 0.001$ \\
\hline$r_{2}($ pole $)$ & $0.394 \pm 0.001$ & $0.301 \pm 0.001$ \\
\hline$r_{2}($ side $)$ & $0.416 \pm 0.001$ & $0.317 \pm 0.001$ \\
\hline$r_{2}($ back $)$ & $0.447 \pm 0.002$ & $0.367 \pm 0.003$ \\
\hline$r_{2}$ (average) & $0.419 \pm 0.001$ & $0.328 \pm 0.002$ \\
\hline$f$ & $1.99( \pm 0.01) \%$ & $41.04( \pm 0.07) \%$ \\
\hline$a\left(R_{\odot}\right)$ & $2.05 \pm 0.14$ & $2.44 \pm 0.23$ \\
\hline$V_{\gamma}\left(\mathrm{km} \mathrm{s}^{-1}\right)$ & $-0.09 \pm 0.31$ & $\cdots$ \\
\hline$M_{1}\left(M_{\odot}\right)$ & $0.69 \pm 0.04$ & $0.84 \pm 0.10$ \\
\hline$M_{2}\left(M_{\odot}\right)$ & $1.07 \pm 0.06$ & $0.33 \pm 0.04$ \\
\hline$R_{1}\left(R_{\odot}\right)$ & $0.70 \pm 0.05$ & $1.19 \pm 0.11$ \\
\hline$R_{2}\left(R_{\odot}\right)$ & $0.86 \pm 0.06$ & $0.80 \pm 0.08$ \\
\hline$L_{1}\left(L_{\odot}\right)$ & $0.20 \pm 0.01$ & $1.01 \pm 0.20$ \\
\hline$L_{2}\left(L_{\odot}\right)$ & $0.24 \pm 0.01$ & $0.44 \pm 0.08$ \\
\hline$\sum \omega_{i}(O-C)_{i}^{2}$ & 0.8444 & 2.1410 \\
\hline Chi squared & 0.9813 & 0.9253 \\
\hline
\end{tabular}

temperature: $3636 \mathrm{~K} \pm 323 \mathrm{~K}$. The $\mathrm{W}-\mathrm{D}$ program is very normal and is useful for obtaining the parameters of eclipsing binaries. The solution with the two-spot model outperforms the other models that we have tried, but does not completely fit the data. Perhaps this suggests the surface distribution for V582 Lyr is more complicated than what you are modeling currently. 

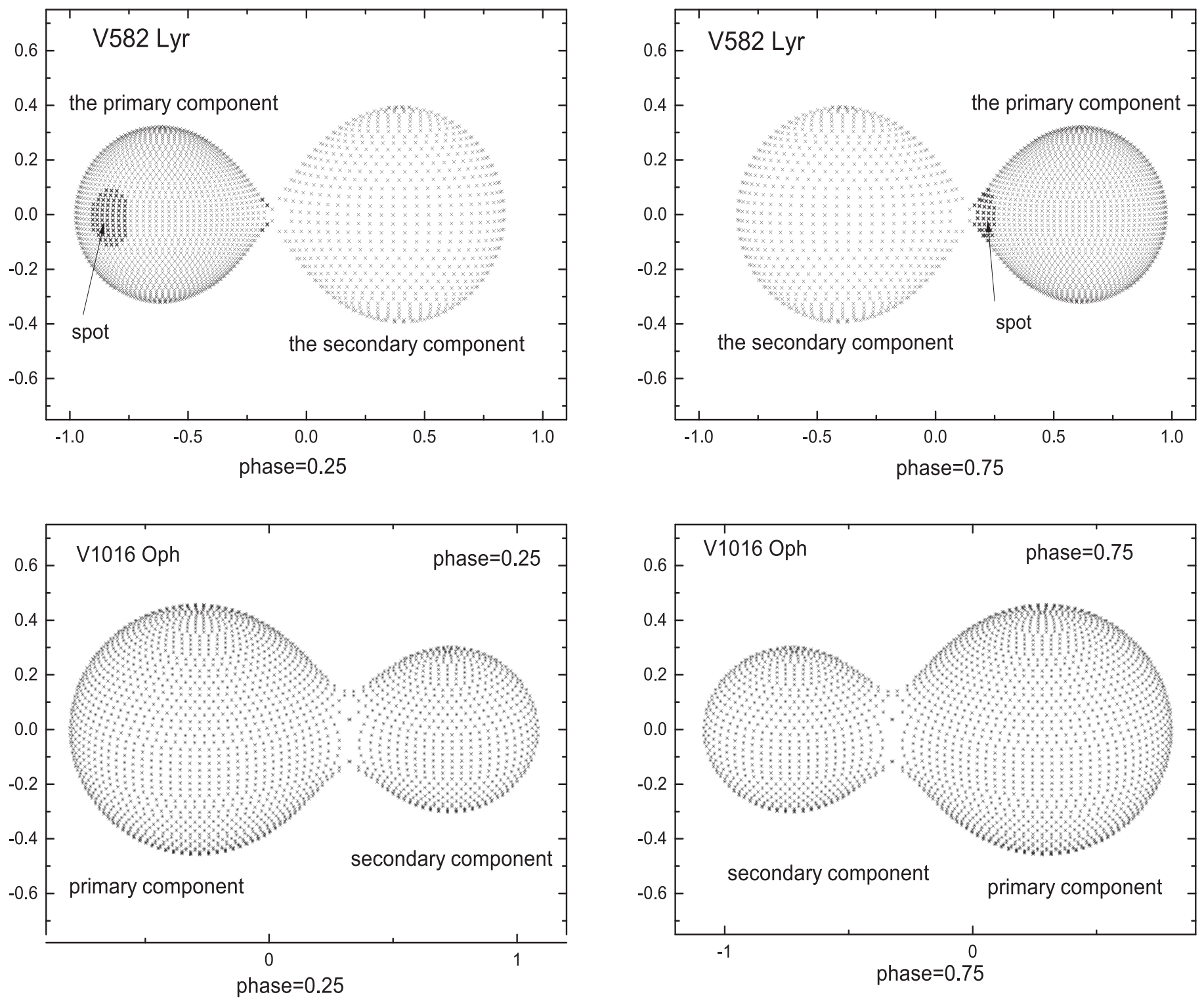

Figure 8. Configuration and spot distribution of V582 Lyr and V1016 Oph.

A disadvantage of the $\mathrm{W}-\mathrm{D}$ program is that they have many free parameters and the shapes of spots or spot distributions have to be assumed (Berdyugina 2005). We believe that if we consider a spot temperature distribution and covering fraction, the fit will be better.

From Figure 1, the model light curves from our WilsonDevinney fitting for V582 Lyr still do not match the data very well. The shortcoming of the W-D program is that we do not consider a spot temperature distribution and covering fraction (Lanza et al. 1998). We will reduce our light curve using the inversion techniques based on the Maximum Entropy and Tikhonov regularizations (Rodono et al. 1995; Lanza et al. 1998). We assumed the spot shape is a circle and the temperature is the same in the circle of one spot (Wilson 1979). Their errors were computed from the W-D differential correction program, and are smaller than the real errors. The Wilson-Devinney program does not provide error estimates using the Markov Chain Monte Carlo method (Boffin et al. 2018).

According to the spectra type of V1016 Oph, we determined its effective temperature to be about $5310 \mathrm{~K}( \pm 160)$, which was chosen as the initial value. The parameters of the bolometric albedo and gravity-darkening coefficients were set the same way to V582 Lyr. The bolometric limb-darkening coefficients $X_{\text {bolo }}$ and limb-darkening coefficients for $B V R I$ bands are $X_{1 \text { bolo }}$ $=0.522, X_{\text {2bolo }}=0.537 ; X_{1 B}=0.791, X_{2 B}=0.864, X_{1 V}$ $=0.654, X_{2 V}=0.725, X_{1 R}=0.541, X_{2 R}=0.599, X_{1 I}=0.444$, $X_{2 I}=0.489$. We found the minimum $\Sigma \omega_{i}(O-C)_{i}^{2}$ when $q=0.39$. After debugging these adjustable parameters to converge, we found the final photometric solution of V1016 Oph. Its corresponding parameters are listed in Table 6. The photometric light curves of these four bands were explained by our photometric solution, and the fitting result is shown in Figure 1. The corresponding model of the stellar structure is presented in Figure 8.

\subsection{Chromospheric Activity of V582 Lyr}

We normalized the spectra of V582 Lyr to their continuum with a six-order polynomial fit using the continuum package in the IRAF software. Because the chromospheric active line $\mathrm{H}_{\alpha}$ is blended with other nearby photospheric lines, we employ a 

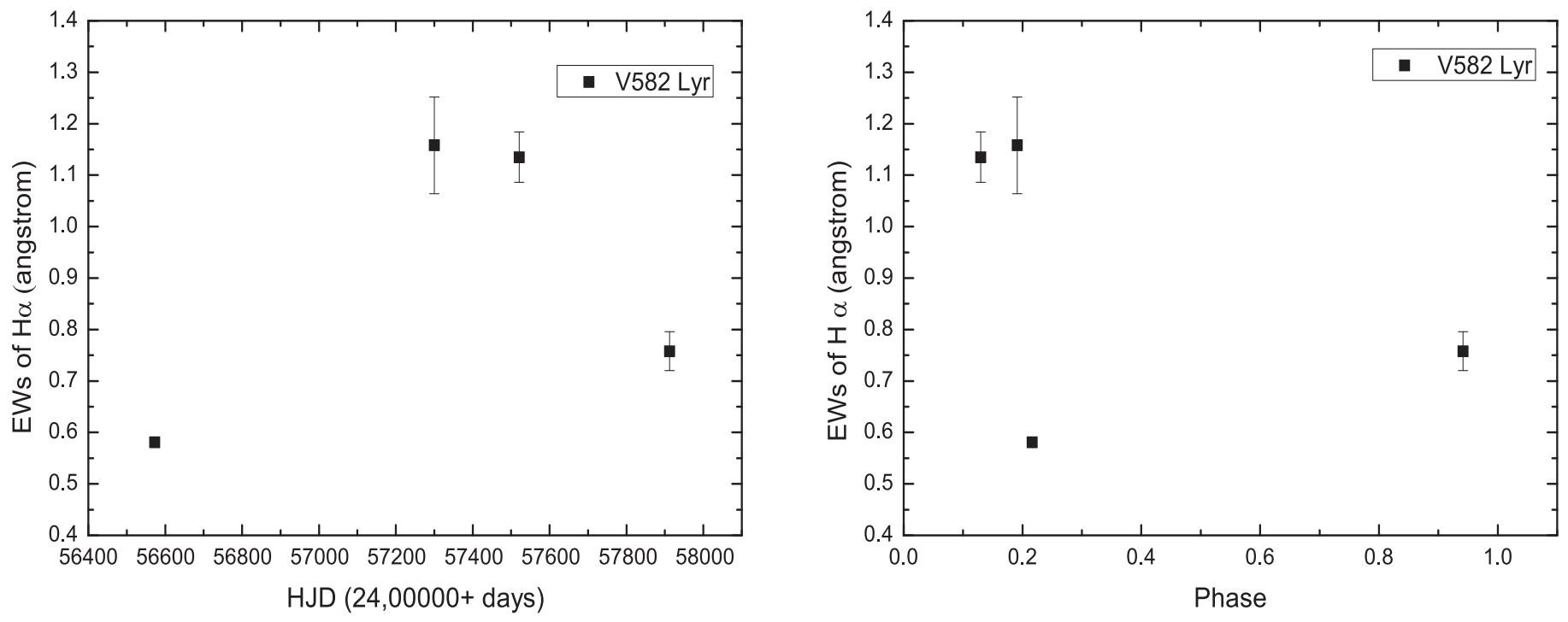

Figure 9. Chromospheric active variations of V582 Lyr vs. HJD (left) or phase (right) in the $\mathrm{H}_{\alpha}$ line.

spectral subtraction technique (Barden 1985) to study the chromospheric activity of V582 Lyr. For the spectral subtraction technique, the synthesized spectra from the photospheric contribution of V582 Lyr were constructed from a rotationally broadened and radial-velocity-shifted inactive star. We downloaded about 10 LAMOST spectra of inactive stars with different spectral types and luminosities from the LAMOST website to serve as candidates for the template. We constructed the synthesized spectra using the inactive spectra. We fit spectra of V582 Lyr spanning wavelength ranges outside the $\mathrm{H}_{\alpha}$ line to obtain the sum of the squared residuals of the fit. We found that HIP20563 is the best template. The spectral type of HIP 20563 was determined to be about K3 $( \pm 2)$ and its temperature was about $4643( \pm 39) \mathrm{K}$, as obtained by the LAMOST survey. The corresponding temperature of V582 Lyr is similar to that of the previous orbital fitting section. We subtracted a synthesized spectrum from the best template to obtain its chromospheric contribution. The observed, subtracted, and synthesized spectra are shown in Figure 2. There was weakH $\mathrm{K}_{\alpha}$ line emission in the subtracted spectra V582 Lyr and weak chromospheric activity. We calculated the equivalent widths (EWs), which express the intensity of the activity, by integrating over the $\mathrm{H}_{\alpha}$ line using the Splot package of the IRAF program.

The EWs are listed in Table 3. We also plot the EWs of the $\mathrm{H}_{\alpha}$ line versus HJD (left) and the orbital phase (right) in Figure 9. That figure shows possible long-timescale variation or variation of different orbital phases in the $\mathrm{H}_{\alpha}$ line.

\section{Discussions and Conclusions}

By calculating the photometric solution using the W-D program with high resolutions and all phase light curves, our photometric solution exhibited the O'Connell effect with not only a hot spot, but we calculated a spot in phase of 0.82 and a hot spot in phase of 0.35 . The photometric solution parameters are as follows: the mass ratio is $1.55( \pm 0.05), 0.05$ is the calculation error in W-D program; the inclination of orbit $i$ is $86.4( \pm 0.3)$ degrees; the percentages of primary component luminosity to total light of V582 Lyr in different ( $B V R I)$ bands were 0.5201 ( \pm 0.0012$), 0.4968( \pm 0.0011), 0.4746( \pm 0.0010)$, and $0.4590( \pm 0.0007)$. The dimensionless potential of V582 Lyr is $\Omega_{1}=\Omega_{2}=4.581( \pm 0.113)$. We calculated a contact factor of V582 Lyr as $3.347( \pm 0.083) \%$.

Based on our LAMOST spectral or color indexes of the Cox relation (Cox 2000), we can assume spectral types. After that, we used our obtained mass ratio to calculate the masses ofsecondary components. According to Kepler's third law $\left(M_{1}+M_{2}=0.0134 a^{3} / p^{2}\right)$, we calculated the semimajor axis $a$. The radii were derived using formula $R_{1,2}=a \times r_{1,2 \text { (mean). }}$. Although the objects we study are contact binaries, we have to use the mean fractional radii from our photometric solutions for $r_{1,2 \text { (mean). }}$ Then, we obtain the luminosity using the equation $L_{1,2}=\left(R_{1,2} / R_{\odot}\right)^{2}\left(T_{1,2} / T_{\odot}\right)^{4}$, where $T_{\odot}=5780 \mathrm{~K}$. We used the error transfer formula to obtain their errors. The masses and radii are the error calculated by different $\mathrm{W}-\mathrm{D}$ correction programs, which are smaller than the real errors. The Wilson Devinney program does not provide an error estimate for orbital parameters using the Markov Chain Monte Carlo method for the eclipsing binaries (Boffin et al. 2018). Combining the photometric solution and the relationship between mass and color index (Cox 2000), we could deduce the primary component mass of V582 Lyr as $M_{1}=0.69 M_{\odot}$ and the secondary component $M_{2}=q \times M_{1}=1.07 M_{\odot}$. The new ephemeris of V582 Lyr was calculated by adding the new minimum light times of observation. It is known from our analyses of the $O-C$ downward parabola curve that the period of V582 Lyr is decreasing (Qian et al. 2005) and the rate of change is $d p / d t=-4.34( \pm 0.13) \times 10^{-8}$ days $\mathrm{yr}^{-1}$, which may mean that there was material moving from the secondary component to another one (Hoffman et al. 2006) or magnetic braking (Applegate 1992; Lanza et al. 1998; etc.). To count how much mass had been transferred, we used the formula (Singh \& Chaubey 1986)

$$
\frac{d p / d t}{P}=3\left(\frac{M_{2}}{M_{1}}-1\right) \frac{d M_{2} / d t}{M_{2}} .
$$

Therefore, for V582 Lyr, we obtained the mass transfer rate $d M_{2} / d t=-1.10( \pm 0.03) \times 10^{-7} M_{\odot} \mathrm{yr}^{-1}$.

For V1016 Oph, the mass of the primary component was determined at $M_{1}=0.84 M_{\odot}$ using the same method, then we 
obtained $M_{2}=0.33 M_{\odot}$ using the mass ratio $q=0.39$. The ratios of the luminosities of the primary component for V1016 Oph were $0.7135 \quad( \pm 0.0013), 0.7099 \quad( \pm 0.0011), 0.7067$ $( \pm 0.0010)$, and $0.7040( \pm 0.0009)$ in different bands $(B V R I)$. The orbital inclination was $71.0 \pm(0.2)$ degrees, and the dimensionless potential was $\Omega_{1}=\Omega_{2}=2.560( \pm 0.005)$. Our method of using the Wilson Devinney program does not provide the error estimate. The period of V1016 Oph is decreasing and the rate is $d p / d t=3.46( \pm 0.05) \times 10^{-7}$ days $\mathrm{yr}^{-1}$, so the rate of mass transfer is $d M_{1} / d t=-2.69( \pm 0.04) \times 10^{-7} M_{\odot} \mathrm{yr}^{-1}$. Finally, we calculated the contact factor of V1016 Oph as $41.0( \pm 0.1) \%$; the analog image displayed in Figure 8 shows that they are closed contact from the model.

The secondary component of V582 Lyr has lower temperature and lower mass, so we could postulate that the two components are similar and their masses are balanced because of the mass transfer. For V1016 Oph, the secondary component has a lower temperature but we could account for most of the mass by attributing it to mass transfer or magnetic activity. More spectroscopic observations are needed to discuss the precise evolutions of V582 Lyr and V1016 Oph.

This research was supported by the Joint Research Fund in Astronomy (No.11963002, U1631236 and U1431114) under cooperative agreement between the NSFC and CAS. We thank Dr. Frank H. Levinson for his generous financial support that enabled Butler University to join SARA and upgrade the Holcomb telescope.

\section{References}

Akerlof, C., Amrose, S., Balsano, R., et al. 2000, AJ, 119, 1901 Applegate, J. H. 1992, ApJ, 385, 621

Armstrong, D. J., Gómez, M. C. Y., Faedi, F., et al. 2014, MNRAS, 437, 3473 Avvakumova, E. A., Malkov, O. Y, \& Kniazev, A. Y. 2013, AN, 334, 860 Barden, S. C. 1985, ApJ, 295, 162

Berdyugina, S. V. 2005, LRSP, 2, 8

Billings, G. W., Kaiser, D. H., Terrell, D., et al. 2001, IBVS, 5029, 1

Blättler, E., \& Diethelm, R. 2000, IBVS, 4985, 1

Boffin, H. M. J., Jones, D., Wesson, R., et al. 2018, A\&A, 619, 84

Brát, L., Zejda, M., \& Svoboda, P. 2007, OEJV, 0074, 1

Conroy, K. E., Prsa, A., Stassun, K. G., et al. 2014, AJ, 147, 45

Cox, A. N. 2000, Allen's Astrophysical Quantities (4th ed.; New York: AIP)

Cui, X. Q., Zhao, Y. H., Chu, Y. Q., et al. 2012, RAA, 12, 1197

Cutri, R. M., Skruskie, M. F., van Dyk, S., et al. 2003, yCat, 2246, 0

Diethelm, R. 2003, IBVS, 5438, 1

Diethelm, R. 2004, IBVS, 5543, 1

Diethelm, R. 2005, IBVS, 5653, 1

Diethelm, R. 2007a, IBVS, 5837, 1

Diethelm, R. 2007b, IBVS, 5781, 1

Diethelm, R. 2009, IBVS, 5894, 1

Diethelm, R. 2010a, IBVS, 5920, 1

Diethelm, R. 2010b, IBVS, 5945, 1

Diethelm, R. 2011, IBVS, 5992, 1
Diethelm, R. 2012, IBVS, 6029, 1

Drake, A. J., Graham, M. J., Djorgovski, S. G., et al. 2014, ApJS, 213, 9

Fan, Y., Bai, J. M., Zhang, J. J., et al. 2015, RAA, 15, 918

George, D. B. 2000, IAPPP, 79, 2

Hoffman, D. I., Harrison, T. E., et al. 2006, AJ, 132, 2260

Hübscher, J. 2017, IBVS, 6196, 1

Hübscher, J., Lehmann, P. B., \& Walter, F. 2012, IBVS, 6010, 1

Keel, W. C., Oswalt, T., Mack, P., et al. 2017, PASP, 129, 5002

Kinman, T. D., Wirtanen, C. A., Janes, K. A., et al. 1965, ApJS, 11, 223

Kirk, B., Conroy, K., Prsa, A., et al. 2016, AJ, 151, 68

Koleva, M., de Rijcke, S., Prugniel, P., Zeilinger, W. W., \& Michielsen, D. 2009a, MNRAS, 396, 2133

Koleva, M., Prugniel, P., Bouchard, A., \& Wu, Y. 2009b, A\&A, 501, 1269

Krajci, T. 2005, IBVS, 5592, 1

Krajci, T. 2006, IBVS, 5690, 1

Kukarkin, B. V., Efremov, Yu. N., Frolov, M. S., et al. 1968, IBVS, 311, 1

Kwee, K. K., \& van Woerden, H. 1956, BAN, 12, 327

Lampens, P., Van Cauteren, P., Ayiomamitis, A., et al. 2017, IBVS, 6230, 1

Lanza, A. F., Catalano, S., Cutispoto, G., et al. 1998, A\&A, 332, 541

Lanza, A. F., Rodonó, M., \& Rosner, R. 1998, MNRAS, 296, 893

Lucy, L. B. 1967, ZA, 65, 89

Luo, A. L., Zhao, Y. H., Zhao, G., et al. 2015, RAA, 15, 1095

Malkov, O. Y., Oblak, E., et al. 2006, A\&A, 446, 785

Massey, P., \& Davis, L. E. 1992, A Users Guide to Stellar CCD Photometry with IRAF, https://www.mn.uio.no/astro/english/services/it/help/ visualization/iraf/daophot2.pdf

Nelson, R. H. 2007, IBVS, 5760, 1

Nelson, R. H. 2011, IBVS, 5966, 1

Nelson, R. H., Terrell, D., \& Milone, E. F. 2014, NewAR, 59, 1

Otero, S. A., Hoogeveen, G. J., \& Wils, P. 2006, IBVS, 5674, 1

Paschke, A. 2009, OEJV, 116, 1

Paschke, A. 2012, OEJV, 142, 1

Paschke, A. 2013, OEJV, 155, 1

Paschke, A., \& Brát, L. 2006, OEJV, 23, 13

Pickles, A. J. 1998, PASP, 110, 863

Prsa, A., Conroy, K. E., Horvat, M., et al. 2016, ApJS, 227, 29

Prsa, A., \& Zwitter, T. 2005, ApJ, 628, 426

Qian, S. B., He, J. J., Zhang, J., et al. 2017, RAA, 17, 87

Qian, S.-B., Yang, Y.-G., Soonthornthum, B., et al. 2005, AJ, 130, 224

Robertson, J. P., \& Eggleton, P. P. 1977, MNRAS, 179, 359

Rodono, M., Lanza, A. F., Catalano, S., et al. 1995, A\&A, 301, 75

Rucinski, S. M. 1973, AcA, 23, 79

Shaw, J. S. 1994, MmSAI, 65, 95

Singh, M., \& Chaubey, U. S. 1986, Ap\&SS, 124, 389

Van Hamme, W. 1993, AJ, 106, 2096

Wang, S. G., Su, D. Q., Chu, Y. Q., et al. 1996, ApOpt, 35, 5155

Wilson, R. E. 1979, ApJ, 234, 1054

Wilson, R. E. 1990, ApJ, 356, 613

Wilson, R. E. 1994, PASP, 106, 921

Wilson, R. E., \& Devinney, E. J. 1971, ApJ, 166, 605

Wilson, R. E., \& Van Hamme, W. 2010, in ASP Conf. Ser. 435, Binaries - Key to Comprehension of the Universe, ed. A. Prša \& M. Zejda (San Francisco, CA: ASP), 45

Wu, Y., Luo, A. L., Li, H. N., et al. 2011a, RAA, 11, 924

Wu, Y., Singh, H. P., Prugniel, P., Gupta, R., \& Koleva, M. 2011b, A\&A, 525, A71

Yang, Y. G., Lu, G. L., Yin, X. G., et al. 2009, AJ, 137, 236

Zhang, L.-Y., \& Gu, S.-H. 2008, A\&A, 487, 709

Zhang, L. Y., Lu, H. P., L. Han, X. M., et al. 2018, NewA, 61, 36

Zhang, L. Y., Yue, Q., Lu, H. P., et al. 2017, RAA, 17, 10

Zhu, L. Y., \& Qian, S. B. 2006, MNRAS, 367, 423 